\title{
What is the Optimal Weight for Gold in a Portfolio?
}

\author{
Brian M. Lucey ${ }^{\mathrm{a}, *}$, Maurice Peat ${ }^{\mathrm{b}}$, Aleksandar Ševićc ${ }^{\mathrm{c}}$, Samuel A. Vigne ${ }^{\mathrm{d}}$ \\ ${ }^{a}$ Trinity Business School, Trinity College Dublin, Dublin 2, Ireland 6 University of Sydney Business School, Sydney, \\ Australia \&5 \\ Institute of Business Research, University of Economics Ho Chi Minh City, 59C Nguyen Dinh Chieu, Ward 6, District \\ 3, Ho Chi Minh City, Vietnam \\ ${ }^{b}$ University of Sydney Business School, Sydney, NSW, Australia \\ ${ }^{c}$ Trinity Business School, Trinity College Dublin, Dublin 2, Ireland \\ ${ }^{d}$ Trinity Business School, Trinity College Dublin, Dublin 2, Ireland
}

\begin{abstract}
We show that the statistical properties of gold are negatively correlated with equities and that including gold in a portfolio will provide diversification benefits. As there is no consensus on the proportion of gold that should be included in a strategic portfolio allocation we propose a visual tool that associates a performance metric with a range of possible asset weighting schemes - a Sharpe ratio response surface. This very surface shows that a target performance metric can be achieved with a large number of different allocations. We further argue that the rebalancing approach based on the surface closest to the benchmark surface under the Hausdorrf distance metric should be selected. Using a data sample between 1990 and 2018, we find that annual rebalancing with a 44-week lookback period achieves the minimum distance from the benchmark surface.
\end{abstract}

Keywords: Gold, Portfolio formation, Asset allocation

JEL Codes : C32, F18, F49

\section{Introduction}

In practice many fund managers use target strategic asset allocations as the basis for the investment strategy of their funds. As an example, Sharpe (2010) outlines the Asset Allocation policy settings for the California Public Employees' Retirement System: 66\% equity, 19\% bonds, 5\% inflation-linked assets, $10 \%$ real estate, and $0 \%$ commodities. But how does an asset manager considering the addition of a new asset class to an existing strategic asset allocation plan goes about deciding on the new weighting scheme? There is no well documented and widely accepted approach to guide practitioners in setting asset allocations. Many authors, such as Estrada (2016), refer to a $60 \%$ stock - $40 \%$ bond allocation as being traditional or balanced. This type of portfolio is implied by the Security Market Line (SML) used in the development of the Capital Asset Pricing Model (CAPM). Different points on the SML will lead to different proportions of the market index (stocks) and the risk-free asset (bonds) in the investors portfolio. Investors' appetite for risk will determine the portfolio on the SML they invest in, which also gives their asset allocation. Indeed, Sharpe (2010) argues that in determining an asset allocation policy, an estimates of the risks, returns and correlations of the asset classes should be considered. Any constraints on asset holdings specific to the fund that will implement the allocation should also be taken into account. This process is often a qualitative exercise. There is an extensive literature on the topic of rebalancing a portfolio to keep to target asset allocations, see as examples

\footnotetext{
* Corresponding Author

Email addresses: Email: blucey@tcd.ie (Brian M. Lucey), Email:maurice.peat@sydney.edu.au (Maurice Peat), Email:a.sevic@tcd.ie (Aleksandar Šević), Email:s.vignes@tcd.ie (Samuel A. Vigne)
} 
Sharpe (2010), Chaves et al. (2011), Perold and Sharpe (1995), Allevi et al. (2019), and Asano and Osaki (2019).

In this manuscript, we develop a visualisation of the output from a range of constrained portfolio construction problems, which we describe as a Sharpe ratio response surface. This visualisation clearly shows the impact of differing asset allocations on a return per unit risk basis. Fund managers can use the slope and curvature of the response surface to understand the impact of changes to an existing allocation and the effect of adding a new asset to an existing allocation. The specific problem investigated is the addition of a new asset class, gold, to an existing portfolio of stocks and bonds.

\section{Previous research on gold}

Despite the importance of gold as an investment asset, there is a (relative) paucity of discussion around many aspects of its usage. One aspect that has so far eluded published academic research is the optimal weight of gold in a well-diversified portfolio when asset allocation is dynamic. How does the lookback period affect this? What of short selling and weight constraints? From Keller et al. (2015) we find that in traditional asset portfolios shorter lookback periods and industry related weights greatly improve mean-variance based portfolios. How does gold perform in a mean-variance versus a $1 / N$ portfolio allocation, with varying lookback periods? Does adding gold affect the alleged superiority of a $1 / N$ allocation (Faber (2007), DeMiguel et al. (2007))?

Much of the recent research has revolved around whether or not gold might be considered a hedge or a safe haven. Gold offers an effective hedge against realised inflation both in the short and longterm, and it follows the patterns of the unexpected inflation in the UK, the US, Japan and Switzerland (Conlon et al. (2018)). Baur and Lucey (2010) and Sumner et al. (2010) examine real returns and volatility spillovers between gold, stocks and bonds in the US. They find very little in the way of spillovers to gold from US stocks and bonds in their sample from January 1970 to April 2009. This, it would assume, is the (lack of a channel) that allows gold to be seen as a significant diversifying force. So this raises the question of the extent to which gold should be part of a portfolio. By extending the study on a sample of developing and emerging economies, Gürgün and Ünalmi (2014) find that gold serves domestic investors as a hedge and a safe haven in a majority of sampled countries. The benefits for foreign investors are comparatively smaller. By contrast, Bekiros et al. (2017) resort to a non-linear wavelet-copula approach and claim that gold is a good diversifier in BRIC countries, but not as effective as a hedge or a save haven. Some stock-like features of gold are also supported for the Chinese and Indian markets (Bouri et al. (2017)). In addition, stocks, as opposed to gold and oil, have a stronger impact on financial stress, but the reverse causality by financial stress is the weakest on public markets (Das et al. (2018)). When having a look at a more diverse commodity spectrum, Bekiros et al. (2017) examine the relationship between commodity futures and the US equity market by referring to the complex network theory, and conclude that commodity futures have strong relations within the same category. As far as gold is concerned the relevant network is goldplatinum-copper-platinum. However, the link between gold and equity is rather weak. Conversely, the link between gold futures and spot prices is unsurprisingly strong (Jena et al. (2018)). There is a routine comparison of gold with the most dominant cryptocurrencies in terms of valuation that originated in the blockchain ledger technology. While Dyhrberg (2016a) claims that bitcoin may be 
located somewhere between gold, as a store value, and the US currency, and that it could be used as a hedging instrument, Dyhrberg (2016b), Demir et al. (2018)), and Baur et al. (2018) claim that crypto products are different from both assets, when risk-return, volatility and relevant correlations have been examined. But if someone invests in bitcoin it seems viable to eventually use gold as a more effective hedging tool than an S\&P 500 composite or wheat (Pal and Mitra (2019)).

Gold is a volatile asset when held alone. Jaffe (1989) recommends holding approximately $10 \%$ of a (stock) portfolio in gold in order to achieve an optimal allocation. This however works only with the actual asset. Adding a portfolio of gold stocks, however, is found to increase risk and return in contrast, which, while it may increase the risk-reward ratio overall, does not have the double benefit of simply adding gold.

Using a monthly data set over a longer period (1971 to 1988) Chua et al. (1990)) confirm that gold has a low beta, as per the CAPM, and find that it is consistently insignificantly different from zero across different time periods. This shows that gold price movements have no correlation with stock price movements on average over the period examined. This is what gives gold the ability to hedge portfolio risk. Gold stocks are again shown as a poor diversifier relative to gold and to have a time varying beta in contrast to gold's stable beta.

Another characteristic that contributes to gold's diversification abilities is the skewness of its returns distribution. Lucey et al. (2006) discuss the importance of considering the moments of a distribution, rather than just mean and variance, as is the original portfolio theory. Over the period examined (1988 to 2003) they show that when the positive skew of gold is taken into consideration in a multimoment asset allocation the optimal portfolio weights for gold are lower than under a simple mean-variance analysis. It is recommended that investors should hold between 4 to $6 \%$ gold under traditional optimisation and 2 to $4 \%$ when skewness is accounted for. Surprisingly little research has been undertaken on multimoment optimisation on precious metals.

Hillier et al. (2006) examine the roles of gold, silver and platinum in asset allocation decisions. The paper concludes that all three precious metals provided considerable diversifying benefits in volatile markets when held in a portfolio of U.S. or global stocks, while the diversifying role of precious metals is limited during poor market return periods. The authors then consider two strategies to examine the efficiency of their portfolios: a buy-and-hold strategy and a switching strategy with portfolio efficiency measured as the relative reward-to-risk ratio. Their results suggest that in a passive buy-and-hold strategy, the optimal weight of gold in broad-based international equity portfolios is approximately $9.5 \%$, significantly higher than the level of gold found in most funds equity portfolios. It is also higher than any of the estimates of Bruno and Chincarini (2010). The switching strategy for gold, silver and platinum does not provide significant efficiency gains. Bruno and Chincarini (2010) look at optimally weighted portfolios to assess how much gold investors in various countries should have in order to maximise their risk return profile. These vary considerably, with weights from $0.1 \%$ to $12 \%$.

Although primarily concerned with the longterm relationship of gold prices and inflation, Erb and Harvey (2013) examine the asset allocation problem. They investigate the claim that gold is, for institutional investors, "underowned" and suggest (based on various macroeconomic and tactical guides and data) that $2 \%$ might be a useful allocation. They then discuss the practical implications of this allocation. 
One recent work is Emmrich and McGroarty (2013) who update Jaffe (1989) using monthly data from 1981 to 2011. They reinforce the finding that the addition of gold to a range of portfolios reduces the portfolio volatility in all periods examined, the main aim of a diversifying asset. However, poor gold returns in the 1980s and 1990s mean that risk adjusted returns, as measured by the Sharpe and Treynor ratios, still suffer. The paper also shows that the skew of gold returns moderates the effect of negative skewness, as previously highlighted by Lucey et al. (2006), from other assets such as equities when combined in a portfolio. The changes in the benefits to holding gold prompt the authors to suggest that the ability to switch at the correct time into and out of gold would be beneficial. This fact was disputed by Hillier et al. (2006) who found no benefits from switching, even with hindsight. Gold as a possible hedging asset has also been examined in relation to currency portfolios. Sarafrazi et al. (2014) concern themselves with the European portfolio allocation issue. In a paper similar in spirit to this, Estrada (2016) examines optimal asset allocation of a diversified stock-bond 60-40 portfolio when gold is introduced (as well as other alternative assets). Naive mean-variance over the 1971-2014 period suggests weights, depending on what is being optimised, of between $10 \%$ to $36 \%$. Although not primarily focused on optimal asset allocation, Bredin et al. (2017) do provide a variety of weights. The paper examines the ability of precious metals to mitigate downside risk, in a manner analogous to the Safe Haven argument. Their weights vary between $1 \%$ and $30 \%$, presented without analytical justification.

Capie et al. (2005) examine whether gold can act as a hedge against currency risk, specifically as a hedge against Yen/US Dollar and Sterling/US Dollar exchange rates. They show that its ability to act as a hedge is time-varying based on unpredictable political and economic events. The theory as to why it would be a dollar hedge is similar to its negative relationship to the US Dollar. It seems sensible that when the dollar is losing value investors might exchange their dollars for gold, raising the price of gold on average.

Joy (2011) expanded the sample of dollar pairs to 16 currencies from 1986 to 2008. Using dynamic conditional correlation models he finds that gold was a dollar hedge, and becoming more strongly so, over the 23-year period examined. Reboredo (2013) also finds that gold acts as a dollar hedge using weekly data from 2000 to 2011 for 8 currency pairs. Similar findings of gold as a potentially useful hedge for currencies and inflation are reported in Reboredo and Rivera-Castro (2014), Lucey et al. (2017), and Yang and Hamori (2013) who also examines the safe haven properties of gold for currencies, and Apergis (2014) who shows gold as a useful predictor and hedge for the Australian dollar. Both Ozturk and Acikalin (2008) and Soytas et al. (2009) examined the role of gold as a hedge against the Turkish lira both finding that there is hedge potential.

\section{Data and methodology}

\subsection{Data}

Time series for the S\&P 500 Total Return index (SPX), the Barclays Corporate Bond index(BCBI), the Barclays Treasury Bond Index (BSBI), the Deutsche Bank Muni Pulse index (DMBI) and Gold at a weekly frequency were downloaded from Datastream. The series cover 28 years from 1990 to 2018. The $\log$ return for each period, $r_{t}=\ln \left(P_{t} / P_{t-1}\right)$, is calculated for each of the asset series. 


\subsection{Methodology}

The underlying data used to construct the response surface is derived from the solution of a constrained portfolio optimisation problem for each point on a grid of constraint values. The optimisation problem that is solved is the maximisation of the Sharpe ratio subject to linear constraints. That is:

$$
\operatorname{Max} \frac{\left(x^{\prime} \mu\right)}{\left(x^{\prime} \Sigma^{\prime} x\right)^{1 / 2}}
$$

Subject to:

$$
\begin{gathered}
x^{\prime} 1=1 \\
\sum x_{b} \leq \text { bond maximum } \\
x_{g} \geq \text { gold minimum } \\
x_{i} \geq 0 \forall i
\end{gathered}
$$

The control variables are the portfolio weights $\mathrm{x}_{\mathrm{i}} \cdot \mu$ is the vector of expected asset returns and $\Sigma$ is the covariance matrix of returns. The objective is to select the portfolio weights which maximise the return per unit risk, the Sharpe ratio of the portfolio.

Constraint (2) requires the portfolio weights (asset proportions) to sum to one; the portfolio is fully invested in Gold, Bonds and Equities. Full investment in the three assets is consistent with a fund manager holding cash, as this cash would be invested in some form of short term risk free bond. Constraint (5) is the long only constraint, there are no short positions allowed. The third constraint (3) puts an upper bound on the holding of bonds in the portfolio. The fourth constraint (4) sets a lower bound on the proportion of gold in the portfolio, which has the effect of forcing gold to be held as part of the portfolio.

A Sharpe ratio can be calculated for any portfolio that is constructed. Fund managers considering the asset allocation problem would be interested in the relative performance of a range of potential allocations in terms of their return per unit risk. To allow for a convenient comparison of the performance of a range of possible allocations of three assets, a three dimensional data visualisation is developed. The $\mathrm{X}$ and $\mathrm{Y}$ axes correspond to upper bound on gold and bonds in the portfolio, the $\mathrm{Z}$ axis is the return/risk ratio that corresponds to the optimal portfolio formed using the gold and bond limits. Using this visual tool a fund manager can easily determine the range of possible asset allocations which correspond to a target return/risk score. Solving the optimisation problem over a grid of (bond maximum, gold minimum) pairs provides the data required to construct the surface of Sharpe ratios.

Two approaches to the optimisation problem are employed. In the first the full set of data is employed in a static optimisation for each parameter pair. The second approach rebalances the portfolio using a range of rebalancing frequencies and data lookback options. The rebalancing approach is a better reflection of the approach of most fund managers. Thus the process of defining the weights in a strategic asset allocation is translated to selecting a desired performance outcome from an ordered collection of optimised outcomes, a Meta optimisation. 


\section{Results}

In Table 1 the properties of the asset returns are presented. The average return of stocks and bonds reflects the expected risk return relationship, higher average returns associated with higher risk as measured by the standard deviation. Gold returns display high volatility relative to the lower return that it earns. The Sharpe ratios of the bond indices are all greater than one, with corporate bonds offering the highest return per unit risk over the 28-year sample period. Stocks and gold both have returns per unit risk less than one, reflecting their volatility.

The box plots of the distributions of the weekly asset returns in Figure 1 are useful in gauging the relative volatility (risk) of the assets under consideration. The various bond indices display little variation in comparison to the stock and gold assets. The almost total lack of variability in the municipal bond series is noteworthy. Even though the average return is considerably smaller than for all the other assets, the low standard deviation gives rise to a Sharpe ratio larger than one. These characteristics will lead to this asset dominating unconstrained portfolios formed using risk minimisation or Sharpe ratio maximisation objectives.

The correlations of the assets in Table 2 makes it clear that there will be clear diversification benefits from forming portfolios that include stocks and bonds, which also have negative or small correlations with stocks. Including gold, an asset with negative correlation to stocks, in the portfolio mix at the expense of stocks will also provide diversification benefits, as has been seen in the literature review.

The first step in the process of evaluating a new asset for inclusion in a portfolio is to explore the characteristics of optimised portfolios with and without the new asset; a benchmark is required for such an evaluation. In the first instance these optimisations are carried out without constraints on the weights of assets in the portfolio, but including the long only and full investment constraints. Three optimisation criteria are compared, the first maximises returns, the second minimises variance and the third maximises return per unit risk (the Sharpe ratio). A set of portfolio optimisations which apply a succession of constraints on bonds is then run. The results of these constrained optimisations can be used in the asset allocation to set a strategic portfolio mix which maximises a fund performance criteria. The performance criteria that is used is the Sharpe ratio, a measure of return per unit risk that is commonly used to evaluate the performance of managed funds.

In Table 3 global optimisation results are presented; return maximisation selects only stocks, both the criterion that include a risk element select a portfolio that is effectively a mix of bonds only.

In Table 4 the impact on the Sharpe ratio and returns of constraining the proportion of bonds in the optimising portfolio is presented. The constraint is set as an upper bound on the holding of bonds, as the portfolio result of the unconstrained maximisation is effectively bonds only, the holding of bonds will always be constrained to the bound set. The trade-off between performance and risk is clear.

In deciding on a strategic asset allocation the manager would select a return or a Sharpe ratio target and then use the associated bound on bonds in the portfolio as the target weight for bonds, with one minus this bound being the target weight for stock. For example an asset manager who was targeting a Sharpe ratio of one (1) would set the strategic allocation of assets in their portfolio to $40 \%$ stock, $60 \%$ bonds, the historic annual return for this allocation being $3.26 \%$. For reference, the $60 \%$ 
stock, $40 \%$ bonds balanced allocation has a Sharpe ratio of 0.8 and a historic return of $3.68 \%$.

The outcomes of repeating the bond holding constrained optimisations with annual rebalancing using a 44-week lookback period are presented in Table 5. This approach to managing a portfolio would result in 28 different sets of weights which satisfy the constraints, each set being constructed using the prior years asset return data. Figure 2 shows the evolution of portfolio structure for the $60 \%$ stock $40 \%$ bonds target allocation. The mix of bonds that make up the $40 \%$ bond allocation evolves over time reflecting the relative performance of the various bonds in the preceding year. These results are a realistic view of the outcomes that a fund manager could expect to achieve based on a set of target portfolio weights and static rebalancing policy. The fund manager targeting a Sharpe ratio of one using strategic allocation of $40 \%$ stock and $60 \%$ bonds using annual rebalancing would have achieved a Sharpe ratio of 0.82 and an annual return of $2.38 \%$.

Determining strategic portfolio allocations in the case where a new asset is being considered for inclusion in the portfolio can be seen as an extension of the approach outlined above. A three dimensional surface representation of the performance metric over a grid of bounds on portfolio composition is constructed. Adding gold as an asset that will have a strategic allocation in addition to stocks and bonds in a portfolio will be used to illustrate the approach. Firstly a grid of gold and bond bounds is defined. Upper bounds are set for bonds and lower bounds are set for gold, the weight allocated to stocks is one minus the sum of the other asset weights in this approach. As outlined in the method section, a constrained portfolio optimisation that maximises the Sharpe ratio is performed for each bound pair.

To illustrate the method, the upper limit on bonds ranges from $20 \%$ to $100 \%$ in $2.5 \%$ increments, while the lower limit on gold ranges from 0 to $50 \%$ in $2.5 \%$ increments. Figure 3 shows a response surface of maximal Sharpe ratios computed with no portfolio rebalancing and the contour map that corresponds to this surface. These visualisations show the range of strategic portfolio weights that can achieve a desired Sharpe ratio. If a fund manager had a Sharpe ratio of one as a target, the green band on the response surface and contour map contains the bounds on bonds and gold that can achieve the objective. It is clear that there are a large number of possible allocations that are capable of achieving the manager's objective. With the proportion of bonds in the portfolio set at $50 \%$ any allocation to gold between $0 \%$ and $30 \%$ will achieve the target Sharpe ratio of one. These regions on the response surface with common performance outcomes present a challenge for managers constructing strategic allocations. The methodical application of constrained optimisation methods does not provide a single optimal allocation, it provides a tool for managers to explore the range of possible strategic allocation that can achieve the performance objective of the fund.

Once a strategic allocation has been chosen, fund managers will engage in a periodic process of adjusting the holdings of assets to account for changes in relative asset values, i.e. rebalancing. Having generated the objective response surface it is possible to find the rebalancing approach which minimises the distance between the response surfaces generated using a range of rebalancing strategies and the surface used in making the initial strategic weights recommendation. The response surface for an annual rebalancing with a 52-week lookback period for portfolio weight estimation is shown in Figure 4. The surface has substantial differences to the benchmark surface, particularly in the region of low proportions of bonds and gold, the lower right section. For illustrative purposes the Sharpe ratio 
surface generated from portfolios formed without rebalancing will be used as the target. Alternative response surfaces will then be formed by generating a Sharpe ratio for portfolios with bond and gold limits that are rebalanced annually with lookback periods of one, five and ten years and quarterly with lookback periods of 3 months, 6 months and 12 months. Finding the alternative which minimises the Hausdorff distance ${ }^{1}$ between its response surface and the benchmark surface provides a quantitative basis for selecting an appropriate rebalancing framework to support a strategic asset allocation. The Hausdorff distance measures the mismatch between two sets (or portions of sets) at fixed positions with respect to each other. It is commonly used in computer vision where it is used to solve the problem of matching a model to an image. In our application the benchmark surface is the model and the surface with rebalancing plays the part of the image.

The results in Table 6 show that the rebalancing approach whose performance is closest to the benchmark surface involves annual rebalancing with a 52 -week lookback period. The quarterly rebalancing approaches generate results that are more volatile than annual approaches, leading to a broader range of computed Sharpe ratios and greater distance from the benchmark response surface. Having established the objective of minimising the distance between response surfaces, the results in Table 6 indicate that an optimal rebalancing approach can be determined.

A binary coded Genetic Algorithm based optimiser is employed to solve this optimisation problem (see Simon (2013) for an introductory exposition of Evolutionary Optimisation methods). The decision variables are the rebalancing frequency and the amount of historical data employed in the portfolio optimisation at each rebalancing point. Three possible rebalancing frequencies are used; annual, quarterly and monthly. Lookback periods from 4 weeks to 511 weeks are considered. These characteristics are encoded onto 11 bits; the rebalancing frequency is represented in the first 2 bits and lookback length is encoded onto the last 9 bits. This encoding allows the discrete nature of the decision variables to be effectively managed in the optimisation. The fitness measure (objective value) to be minimised is the Hausdorff distance between the benchmark Sharpe ratio response surface with no rebalancing and the surface constructed using the candidate solution. The optimisation uses linear rank-based fitness assignment, a single crossover point and uniform random binary mutation. A rebalancing approach which employs annual rebalancing with 44 weeks of historical data as the lookback is found to be optimal.

\section{Conclusion}

The statistical properties of the assets considered in this study show that gold is negatively correlated with equities. This negative correlation supports the argument that including gold in a portfolio will provide diversification benefits. Including a new asset class in strategic portfolio means that the fund manager has to create a new set of target weights that includes the new asset. There is no consensus in the literature on the proportion of gold that should be included in a strategic portfolio allocation. To assist decision makers choosing strategic allocation weights we construct a visual tool

\footnotetext{
${ }^{1}$ The 'directional' Hausdorff distance (dhd) between two sets of points, $\mathrm{P}$ and $\mathrm{Q}$ is defined as: $\operatorname{dhd}(\mathrm{P}, \mathrm{Q})=\max \mathrm{p}$ in $\mathrm{P}[\min \mathrm{q}$ in $\mathrm{Q}[\|\mathrm{p}-\mathrm{q}\|]]$

Intuitively dhd finds the point $\mathrm{p}$ from the set $\mathrm{P}$ that is farthest from any point in $\mathrm{Q}$ and measures the distance from $\mathrm{p}$ to its nearest neighbour in Q. The Hausdorff Distance is defined as $\max (\operatorname{dhd}(\mathrm{P}, \mathrm{Q})$, dhd $(\mathrm{Q}, \mathrm{P}))$.
} 
that associates a performance metric with a range of possible asset weighting schemes; a Sharpe ratio response surface. The surface constructed by maximising the Sharpe ratio without rebalancing provides the benchmark surface used in this study. This surface shows that a target performance metric can be achieved with a large number of different allocation, a finding that challenges the concept of a single optimal allocation. Having selected target weights that can achieve the desired performance criteria a portfolio rebalancing approach is generally applied. There are a large number of rebalancing frequencies and data lookback periods to choose from. The sharpe ratio's evolve smoothly. A response surface over the constraint grid used in the allocation step is constructed for each rebalancing approach being considered. We argue that the rebalancing approach with the surface that is closest to the benchmark surface under the Hausdorrf distance metric should be selected. The rebalancing approach associated with this surface will minimise deviation from the benchmark performance measures. Using our data set we find that annual rebalancing with a 44-week lookback period minimised the distance between surfaces. 


\section{Bibliography}

Allevi, E., L. Boffino, M. E. De Giuli, and G. Oggioni (2019). Analysis of long-term natural gas contracts with vine copulas in optimization portfolio problems. Annals of Operations Research 274 (1-2), $1-37$.

Apergis, N. (2014). Can gold prices forecast the Australian dollar movements? International Review of Economics and Finance 29, 75-82.

Asano, T. and Y. Osaki (2019). Portfolio allocation problems between risky and ambiguous assets. Annals of Operations Research2 Forthcoming.

Baur, D. G., T. Dimpfl, and K. Kuck (2018, June). Bitcoin, gold and the US dollar - A replication and extension. Finance Research Letters 25, 103-110.

Baur, D. G. and B. M. Lucey (2010, May). Is gold a hedge or a safe haven? An analysis of stocks, bonds and gold. Financial Review 45(2), 217-229.

Bekiros, S., , S. Boubaker, D. K. Ngyuen, and G. S. Uddin (2017, May). Black swan events and safe havens: The role of gold in globally integrated emerging markets. Journal of International Money and Finance 73, 317-334.

Bekiros, S., D. K. Nguyen, L. Sandoval Junior, and G. S. Uddin (2017). Information diffusion, cluster formation and entropy-based network dynamics in equity and commodity markets. European Journal of Operational Research 256(3), 945-961.

Bouri, E., D. Roubaud, R. Jammazi, and A. Assaf (2017, Nov). Uncovering frequency domain causality between gold and the stock markets of China and India: Evidence from implied volatility indices. Finance Research Letters 23, 23-30.

Bredin, D., T. Conlon, and V. Potì (2017). The price of shelter - Downside risk reduction with precious metals. International Review of Financial Analysis 49, 48-58.

Bruno, S. and L. Chincarini (2010). A historical examination of optimal real return portfolios for non-us investors. Review of Financial Economics 19(4), 161-178.

Capie, F., T. C. Mills, and G. Wood (2005, Oct). Gold as a hedge against the dollar. Journal of International Financial Markets, Institutions and Money 15(4), 343-352.

Chaves, D., J. Hsu, F. Li, and O. Shakernia (2011). Risk parity portfolio vs. other asset allocation heuristic portfolios. Journal of Investing 20(1), 108-118.

Chua, J. H., G. Sick, and R. S. Woodward (1990). Diversifying with gold stocks. Financial Analysts Journal 46(4), 76-79.

Conlon, T., B. M. Lucey, and G. S. Uddin (2018). Is gold a hedge against inflation: A wavelet time-scale perspective. Review of Quantitative Finance and Accounting 51(2), 317-345. 
Das, D., S. B. Kumar, A. K. Tiwari, M. Shahbaz, and H. M. Hasim (2018, Dec). On the relationship of gold, crude oil, stocks with financial stress: A causality-in-quantiles approach. Finance Research Letters 27, 169-174.

DeMiguel, V., L. Garlappi, and R. Uppal (2007). Optimal versus naive diversification: How inefficient is the 1/n portfolio strategy? The review of Financial studies 22(5), 1915-1953.

Demir, E., G. Gozgor, C. K. M. Lau, and S. A. Vigne (2018, Sep). Does economic policy uncertainty predict the Bitcoin returns? An empirical investigation. Finance Research Letters 26, 145-149.

Dyhrberg, A. H. (2016a, Feb). Bitcoin, gold and the dollar - A GARCH volatility analysis. Finance Research Letters 16, 85-92.

Dyhrberg, A. H. (2016b, Feb). Hedging capabilities of bitcoin. Is it the virtual gold? Finance Research Letters 16, 139-144.

Emmrich, O. and F. McGroarty (2013). Should gold be included in institutional investment portfolios? Applied Financial Economics 23(19), 1553-1565.

Erb, C. B. and C. R. Harvey (2013). The golden dilemma. Financial Analysts Journal 69(4), 10-42.

Estrada, J. (2016). Alternatives: How? How much? Why? Journal of Wealth Management 19(3), 49-61.

Faber, M. T. (2007). A quantitative approach to tactical asset allocation. The Journal of Wealth Management 9(4), 69-79.

Gürgün, G. and I. Ünalmi (2014). Is gold a safe haven against equity market investment in emerging and developing countries? Finance Research Letters 11(4), 341-348.

Hillier, D., P. Draper, and R. Faff (2006, Mar). Do precious metals shine? An investment perspective. Financial Analysts Journal 62(2), 98-106.

Jaffe, J. F. (1989). Gold and gold stocks as investments for institutional portfolios. Financial Analysts Journal 45(2), 53-59.

Jena, S. K., A. K. Tiwari, and D. Roubaud (2018, Mar). Comovements of gold futures markets and the spot market: A wavelet analysis. Finance Research Letters 24, 199-220.

Joy, M. (2011). Gold and the us dollar: Hedge or haven? Finance Research Letters 8(3), 120-131.

Keller, W., A. Butler, and I. Kipnis (2015). Momentum and Markowitz: A Golden Combination.

Lucey, B. M., S. S. Sharma, and S. A. Vigne (2017). Gold and inflation(s) A time-varying relationship. Economic Modelling 67, 88-101.

Lucey, B. M., E. Tully, and V. Poti (2006). International portfolio formation, skewness and the role of gold. Frontiers in Finance and Economics 3(1), 49-68. 
Ozturk, F. and S. Acikalin (2008). Is gold a hedge against turkish lira? South East European Journal of Economics and Business 3(1), 35-40.

Pal, D. and S. K. Mitra (2019, Sep). Hedging bitcoin with other financial assets. Finance Research Letters 30, 30-36.

Perold, A. F. and W. F. Sharpe (1995). Dynamic strategies for asset allocation. Financial Analysts Journal 51(1), 149-160.

Reboredo, J. C. (2013). Is gold a safe haven or a hedge for the us dollar? Implications for risk management. Journal of Banking and Finance 37(8), 2665-2676.

Reboredo, J. C. and M. A. Rivera-Castro (2014, Apr). Can gold hedge and preserve value when the us dollar depreciates? Economic Modelling 39, 168-173.

Sarafrazi, S., S. Hammoudeh, and P. AraújoSantos (2014). Downside risk, portfolio diversification and the financial crisis in the euro-zone. Journal of International Financial Markets, Institutions and Money 32, 368-396.

Sharpe, W. F. (2010). Adaptive asset allocation policies. Financial Analysts Journal 66 (3), 45-59.

Simon, D. (2013). Evolutionary optimization algorithms. John Wiley Sons.

Soytas, U., R. Sari, S. Hammoudeh, and E. Hacihasanoglu (2009, Dec). World oil prices, precious metal prices and macroeconomy in Turkey. Energy Policy 37(12), 5557-5566.

Sumner, S., R. Johnson, and L. Soenen (2010). Spillover effects among gold, stocks, and bonds. Journal of Centrum Cathedra 3(2), 106-120.

Yang, L. and S. Hamori (2013, Dec). Gold prices and exchange rates: a time-varying copula analysis. Applied Financial Economics 24(1), 41-50. 
Table 1: Descriptive Statistics (Full Sample)

\begin{tabular}{llllll}
\hline Stat & SPX & BCBI & BSBI & DMBI & GOLD \\
\hline Mean p.a. & $4.20 \%$ & $2.81 \%$ & $2.35 \%$ & $0.57 \%$ & $1.51 \%$ \\
Sd p.a. & $7.03 \%$ & $2.21 \%$ & $1.96 \%$ & $0.49 \%$ & $6.94 \%$ \\
Sharpe ratio p.a. & 0.597151 & 1.269098 & 1.199072 & 1.174541 & 0.218101 \\
\hline
\end{tabular}

Figure 1: Distributions of Weekly Asset Returns

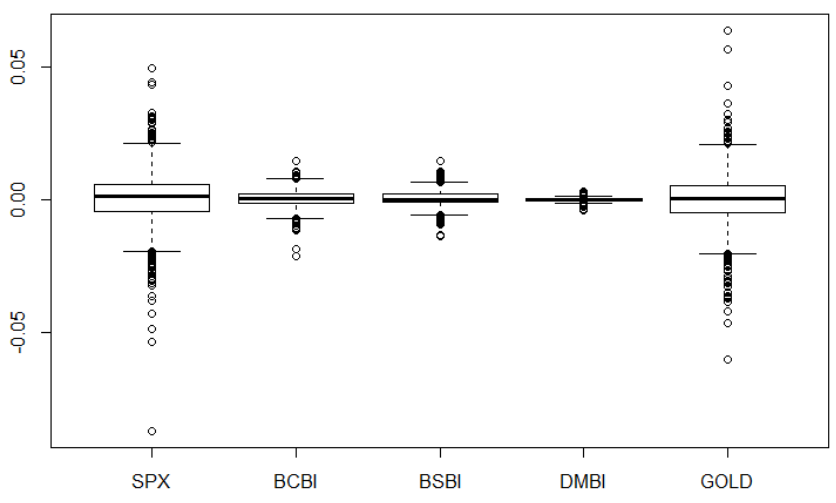

Table 2: Correlation Matrix

\begin{tabular}{lrrrrr}
\hline & SPX & BCBI & BSBI & DMBI & GOLD \\
\hline SPX & 1.000 & 0.014 & -0.176 & -0.086 & -0.027 \\
BCBI & 0.014 & 1.000 & 0.757 & 0.446 & 0.024 \\
BSBI & -0.176 & 0.757 & 1.000 & 0.461 & 0.083 \\
DMBI & -0.086 & 0.446 & 0.461 & 1.000 & 0.054 \\
GOLD & -0.027 & 0.024 & 0.083 & 0.054 & 1.000 \\
\hline
\end{tabular}


Table 3: Base Models, Gold Excluded

\begin{tabular}{lrrr}
\hline & Max Return & Min Variance & Max Sharpe \\
\hline Allocation & & & \\
SPX & 1 & 0.0106 & 0.0524 \\
BCBI & 0 & 0 & 0.0817 \\
BSBI & 0 & 0 & 0.1624 \\
DMBI & 0 & 0.9894 & 0.7036 \\
& & & \\
Performance & & & \\
Return p.a. & $4.21 \%$ & $0.62 \%$ & $1.41 \%$ \\
SD p.a. & $6.99 \%$ & $0.49 \%$ & $0.89 \%$ \\
Sharpe p.a. & 0.60 & 1.32 & 1.59 \\
\hline
\end{tabular}

Table 4: Performance of Base Models with No Rebalancing and a Bound on Bonds

Long Only - No Rebalancing - Upper Bound on Bonds

Max Sharpe Ratio

Bound Sharpe Ratio Return

$20 \% \quad 0.677 \quad 3.94 \%$

$25 \% \quad 0.704 \quad 3.87 \%$

$\begin{array}{lll}30 \% & 0.733 & 3.81 \%\end{array}$

$35 \% \quad 0.767 \quad 3.74 \%$

$\begin{array}{lll}40 \% & 0.805 & 3.68 \%\end{array}$

$\begin{array}{lll}45 \% & 0.847 & 3.58 \%\end{array}$

$\begin{array}{lll}50 \% & 0.896 & 3.47 \%\end{array}$

$\begin{array}{lll}55 \% & 0.953 & 3.36 \%\end{array}$

$\begin{array}{lll}60 \% & 1.021 & 3.26 \%\end{array}$

$\begin{array}{lll}65 \% & 1.101 & 3.16 \%\end{array}$

$\begin{array}{lll}70 \% & 1.192 & 3.06 \%\end{array}$

$\begin{array}{lll}75 \% & 1.293 & 2.96 \%\end{array}$

$\begin{array}{lll}80 \% & 1.393 & 2.88 \%\end{array}$

$\begin{array}{lll}85 \% & 1.438 & 2.47 \%\end{array}$

$\begin{array}{lll}90 \% & 1.481 & 1.86 \%\end{array}$

$\begin{array}{lll}95 \% & 1.568 & 1.40 \%\end{array}$

$\begin{array}{lll}100 \% & 1.596 & 1.42 \%\end{array}$ 
Table 5: Performance of Base Models with Rebalancing and a Bound on Bonds

Long Only - Annual Rebalancing - Upper Bound on Bonds

$\begin{array}{lll}\text { Max Sharpe Ratio } & & \\ \text { Bound } & \text { Sharpe Ratio } & \text { Return } \\ 20 \% & 0.542 & 3.05 \% \\ 25 \% & 0.563 & 2.96 \% \\ 30 \% & 0.591 & 2.90 \% \\ 35 \% & 0.621 & 2.82 \% \\ 40 \% & 0.654 & 2.74 \% \\ 45 \% & 0.690 & 2.65 \% \\ 50 \% & 0.731 & 2.56 \% \\ 55 \% & 0.777 & 2.47 \% \\ 60 \% & 0.827 & 2.38 \% \\ 65 \% & 0.881 & 2.29 \% \\ 70 \% & 0.932 & 2.19 \% \\ 75 \% & 0.959 & 2.02 \% \\ 80 \% & 0.957 & 1.79 \% \\ 85 \% & 0.911 & 1.42 \% \\ 90 \% & 0.825 & 0.96 \% \\ 95 \% & 0.884 & 0.81 \% \\ 100 \% & 1.423 & 1.22 \%\end{array}$

Figure 2: Time Variation of Optimal Weights for 60 - 40 Strategic Allocation

Weights

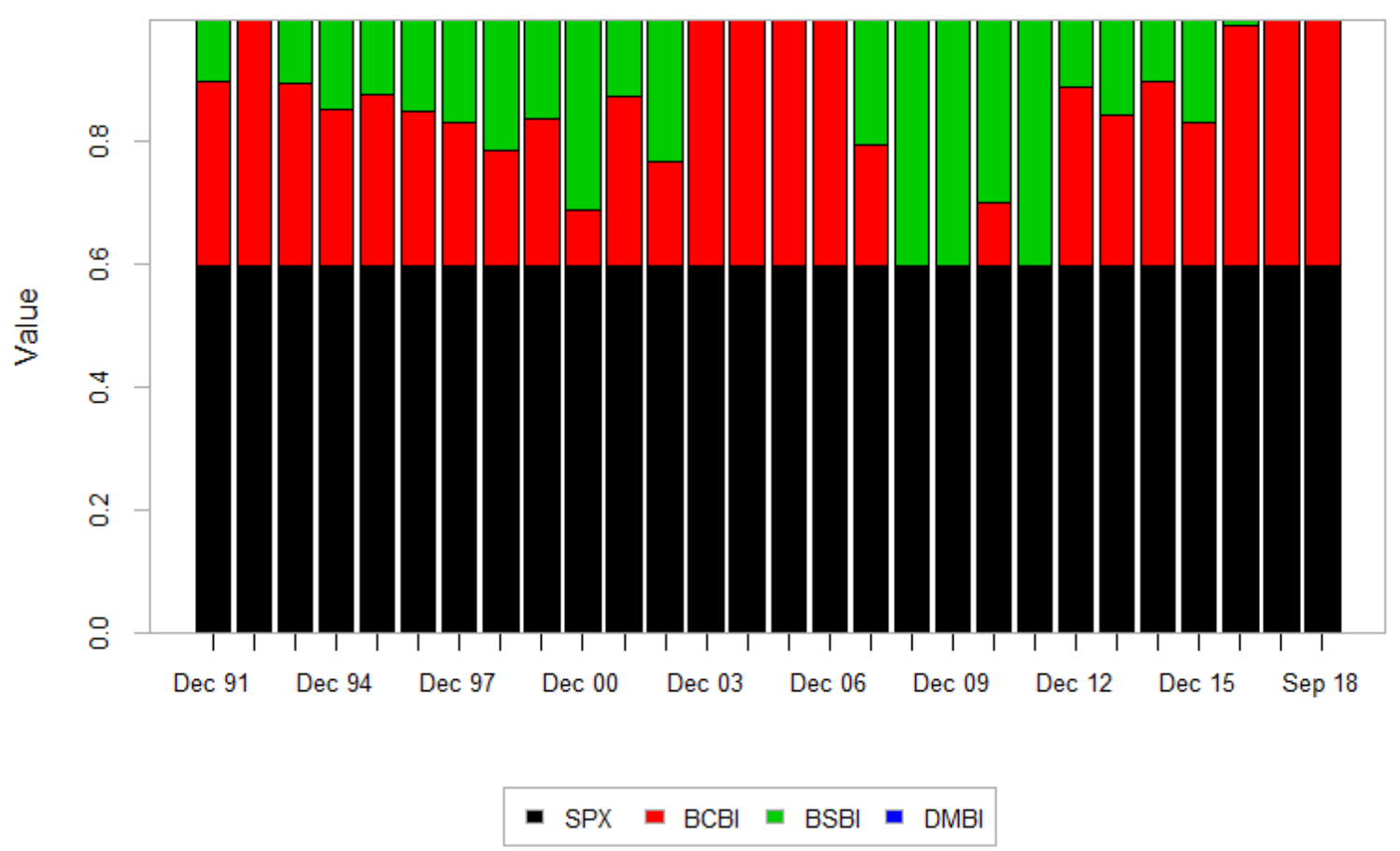


Figure 3: Views of the Sharpe Ratio Response Surface (no Rebalancing) Sharpe Ratio Response Surface

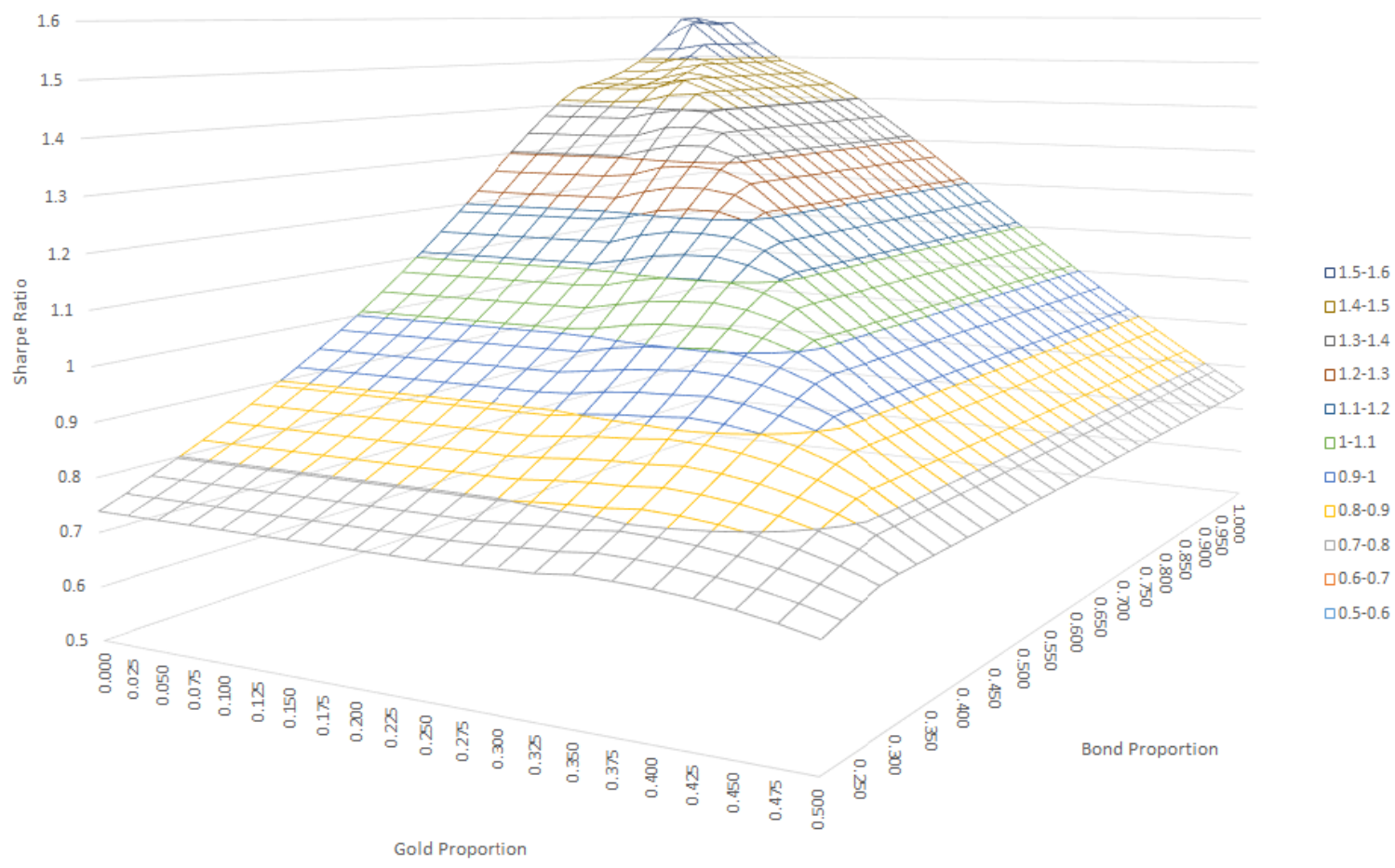

Sharpe Ratio Contour Map

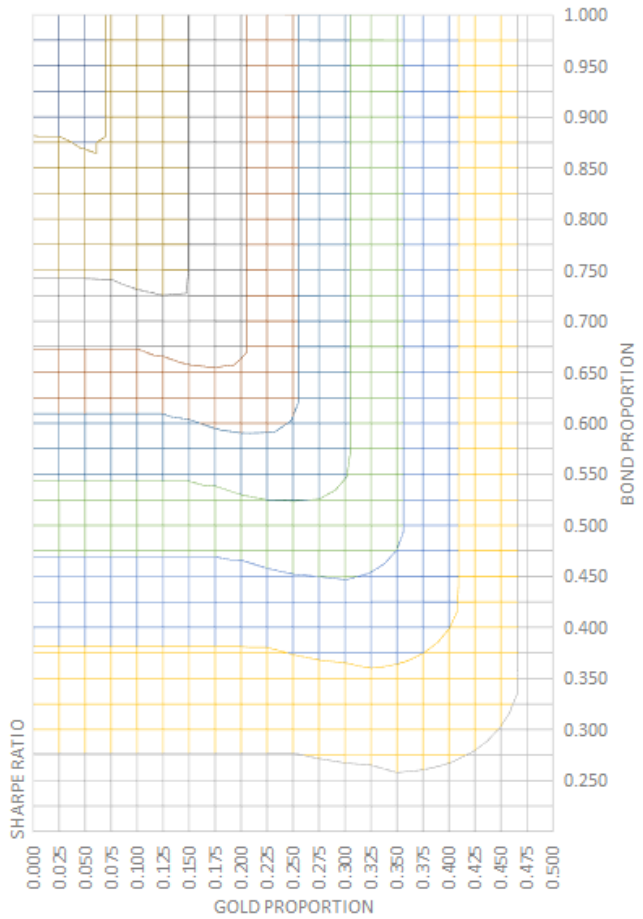


Figure 4: Views of the Sharpe Ratio Response Surface Annual Rebalancing

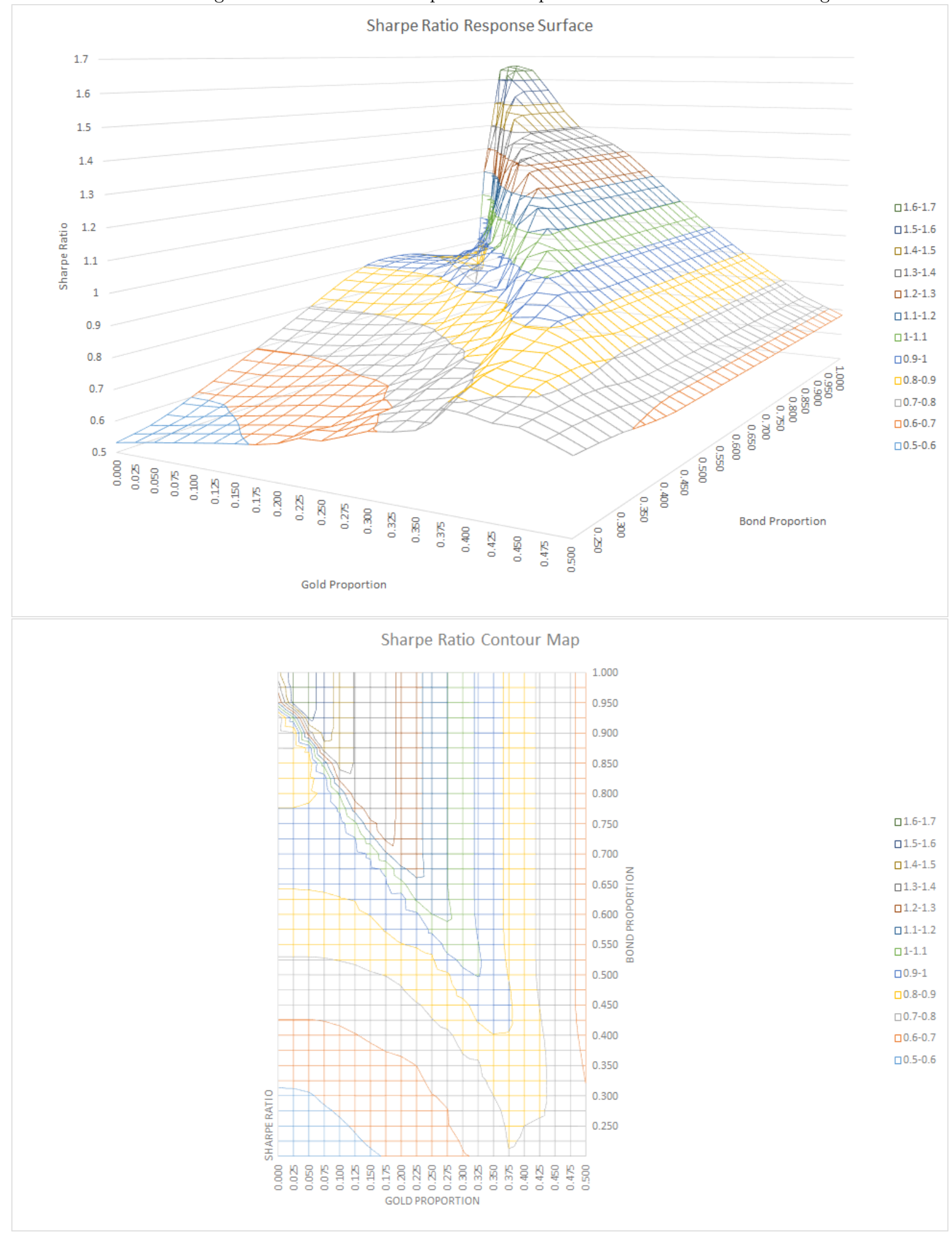


Table 6: Hausdorff Distance from Benchmark Response Surface

Annual Rebalancing

Look back Length Distance

1 Year $\quad 0.8304656$

5 Years $\quad 0.9955918$

10 Years $\quad 1.155948$

Quarterly Rebalancing

\begin{tabular}{ll}
3 Months & 3.537996 \\
6 Months & 3.528482 \\
12 Months & 3.532168 \\
\hline
\end{tabular}

